\title{
Low Doses of Ethanol Reduce Evidence for Nonlinear Structure in Brain Activity
}

\author{
Cindy L. Ehlers, ${ }^{1}$ James Havstad, Dean Prichard, ${ }^{2}$ and James Theiler ${ }^{3}$ \\ ${ }^{1}$ Department of Neuropharmacology, The Scripps Research Institute, La Jolla, California 92037, ${ }^{2}$ Center for Adaptive \\ Systems Applications, Los Alamos, New Mexico 87544, and ${ }^{3}$ Los Alamos National Laboratory, \\ Los Alamos, New Mexico 87544
}

\begin{abstract}
Recent theories of the effects of ethanol on the brain have focused on its direct actions on neuronal membrane proteins. However, neuromolecular mechanisms whereby ethanol produces its CNS effects in low doses typically used by social drinkers (e.g., 2-3 drinks, 10-25 mm, 0.05-0.125 gm/ dl) remain less well understood. We propose the hypothesis that ethanol may act by introducing a level of randomness or "noise" in brain electrical activity. We investigated the hypothesis by applying a battery of tests originally developed for nonlinear time series analysis and chaos theory to EEG data collected from 32 men who had participated in an ethanol/placebo challenge protocol. Because nonlinearity is a prerequisite for chaos and because we can detect nonlinearity more reliably than chaos, we concentrated on a series of measures that quantitated different aspects of nonlinear-
\end{abstract}

The neuromolecular basis of the intoxicating effects of low doses of ethanol is poorly understood. The lipid theory of the actions of alcohol is based on the fact that the behavioral potency of aliphatic $n$-alcohols with up to five carbon atoms is correlated with both their membrane lipid disordering potency and their lipid solubility (membrane/buffer partition coefficient) (Seeman, 1972; Trudell, 1977; Goldstein, 1984; Rall, 1990). Recent theories have shifted from a focus on lipids to include a direct interaction with receptor proteins (Weight, 1992; Grant, 1994; Li et al., 1994; Peoples et al., 1996). Although some effects of ethanol on receptor proteins, such as NMDA, have been reported at concentrations produced by pharmacological doses $(50-100 \mathrm{~mm}, 0.25-0.5$ $\mathrm{gm} / \mathrm{dl}$ ) (Peoples and Weight, 1995), the effects are yet to be significant at concentrations produced by doses typically used by social drinkers (e.g., 2-3 drinks, 10-25 mM, 0.05-0.125 gm/dl).

One explanation for the difficulty in demonstrating significant effects of low doses of ethanol on the neuronal level may be that ethanol affects both proteins and lipids; however, it may not produce a clear agonist or antagonist effect but, rather, may introduce an increased level of randomness in neuronal processing. Several studies provide data that are descriptively supportive of this idea. For instance, Aston-Jones et al. (1982) demonstrated that low doses of ethanol, although having no

Received Dec. 8, 1997; revised June 19, 1998; accepted June 25, 1998.

This work was supported by Grants AA00223, 09652, 04620, and GCRC RR00833 from the National Institute of Alcoholism and Alcohol Abuse. We thank Dr. Marc Schuckit for providing part of the data for analyses, David Cloutier for help in statistics, and Susan Lopez for editing.

Correspondence should be addressed to Dr. Cindy L. Ehlers, The Scripps Research Institute, 10550 North Torrey Pines Road, CVN-14, La Jolla, CA 92037.

Copyright (C) 1998 Society for Neuroscience $0270-6474 / 98 / 187474-13 \$ 05.00 / 0$ ity. For each of these measures the method of surrogate data was used to assess the significance of evidence for nonlinear structure. Significant nonlinear structure was found in the EEG as evidenced by the measures of time asymmetry, determinism, and redundancy. In addition, the evidence for nonlinear structure in the placebo condition was found to be significantly greater than that for ethanol. Nonlinear measures, but not spectral measures, were found to correlate with a subject's overall feeling of intoxication. These findings are consistent with the notion that ethanol may act by introducing a level of randomness in neuronal processing as assessed by EEG nonlinear structure.

Key words: EEG; ethanol; chaos; surrogate data; time series analysis

effect on the mean spontaneous discharge of rat locus coeruleus neurons, significantly increased the variability in the latency at which those neurons fired in response to sensory stimuli. In addition, at the level of the EEG it has been found in both humans and animals that ethanol increases the variance of EEG and event-related potential signal amplitudes over time (Ehlers and Havstad, 1982; Ehlers and Reed, 1987; Ehlers, 1988, 1992; Ehlers et al., 1989).

To assess this hypothesis, we needed to quantitate the effects of ethanol on brain and behavior, using measures that reflect aspects of the dynamical behavior of a system. The present study used a series of statistical measures derived from chaos theory, including measures of time asymmetry, determinism, dimension, and redundancy, to evaluate EEG data collected from 32 subjects participating in an ethanol/placebo challenge protocol. The method of surrogate data, a recent fundamental strategy in nonlinear dynamics, was used to assess the evidence for nonlinearity in these data sets. Using this data set, we explored the following six questions: (1) Is there evidence for significant nonlinear structure in the EEG? (2) Do EEGs collected under a placebo condition differ from those collected after the ingestion of ethanol? (3) Are nonlinear statistics better than linear statistics for distinguishing between EEGs collected under the placebo and ethanol conditions? (4) What measures of nonlinear structure are better in distinguishing an EEG from its surrogates or placebo from ethanol? (5) Does ethanol produce increases or decreases in evidence for nonlinear structure as compared with placebo? (6) Do linear or nonlinear measures of the actions of alcohol on the EEG correlate with a person's subjective report of intoxication? 


\section{MATERIALS AND METHODS}

\section{Subjects}

The subjects who participated in this study were 18 - to 25 -year-old male students and nonacademic staff from the University of California, San Diego. Data were available on men who participated in one of two different ongoing clinical protocols. These subjects met individually with research staff to complete a screening questionnaire (Schuckit and Gold, 1988 ) that was used to select individuals who met the eligibility requirements for the study. Using this highly structured self-report instrument, we excluded subjects from further evaluation if they or their first degree relatives met diagnostic criteria for alcohol or other substance dependence or other major Axis I psychiatric disorders according to the Diagnostic and Statistical Manual, Volume III. The screening questionnaire also was used to gather information on demography, personal medical history, usual quantity and frequency of alcohol consumption over the previous 6 months, and family history of alcohol and other substance dependence. Individuals also were excluded from further study if they were taking prescribed medication, had any major medical condition, or had abstained from alcohol over the previous 6 months. An extensive description of the subject selection process has been described previously (Schuckit, 1985; Ehlers and Schuckit, 1991; Wall et al., 1992, 1993). Some of these men had participated in other parts of larger studies (Ehlers et al., 1989; Ehlers and Schuckit, 1990, 1991; Wall et al., 1992, 1993). Men $(n=32)$ who met the final inclusion criteria then were invited to participate individually in two test sessions, $\sim 1$ week apart, that consisted of baseline evaluations and subsequent challenges with placebo and alcohol.

On both test days each man arrived at the laboratory at approximately 8:00 A.M. after fasting overnight and was provided a standardized low-fat breakfast. Baseline measurements were taken; at approximately 9:00 A.M. a placebo or alcohol beverage was administered in random order, using a placebo alcohol administration device (Mendelson et al., 1984). The alcohol beverage was $0.75 \mathrm{ml} / \mathrm{kg}$ of $95 \%$ alcohol as a $20 \%-$ by-volume solution in a caffeine-free and sugar-free soda. The placebo beverage was made by using the same mixer with $3 \mathrm{ml}$ of $95 \%$ alcohol floated on top. Subjects were instructed to drink at a steady pace and to consume the beverage over $7 \mathrm{~min}$. EEG data were collected at $90 \mathrm{~min}$ after placebo and alcohol. Subjective feelings of intoxication were measured by using a modified version of the Subjective High Assessment Scale (SHAS), which consists of 13 items rated on Likert scales ranging from 0 (normal) to 36 (extreme effect) (see Judd et al., 1977). The total score on this scale was available for each subject and subsequently was used for statistical analyses. Blood samples also were drawn from a heparinized lock inserted into an antecubital vein for subsequent determination of blood alcohol concentrations (BACs), using a modified alcohol dehydrogenase assay.

EEG data were collected from lead P4-02 (right parietal cortex referenced to right occipital cortex), as described previously, because in several studies we have found that lead to be the most sensitive to the effects of ethanol (see Ehlers et al., 1989; Wall et al., 1993). Six minutes of EEG data were collected while the subject was relaxed with eyes closed and with the filters set at $1-70 \mathrm{~Hz}$. The technician carefully monitored the subject for any signs of drowsiness. Three minutes of continuous artifact-free nondrowsy EEG data were computer-analyzed. EEGs were digitized to 12 bits of resolution at 256 samples per second.

All subjects signed informed consent, and the study was approved by the Scripps and University of California, San Diego, internal review boards.

\section{Data analyses}

\section{Conceptual approach to the data analyses}

Nonlinear structure in the EEG after placebo and ethanol administration was quantified by computing a series of measures on the EEG. Most nonlinear measures are sensitive to structure in the data but do not discriminate explicitly between linear and nonlinear structure. Thus to quantify nonlinear structure in the EEG, we constructed a "surrogate" time series from the original EEG signal; the surrogate data mimic the linear structure in the original data, but are otherwise random. Thus, all but the linear structure is effectively removed. In comparing data sets with their associated surrogates, we can identify which have more significant evidence for nonlinear structure. Although we can choose specific measures (e.g., estimated correlation dimension, or in-sample rootmean-square error of a nearest-neighbor prediction algorithm) for quantifying the overall structure in the data, it is difficult to make general
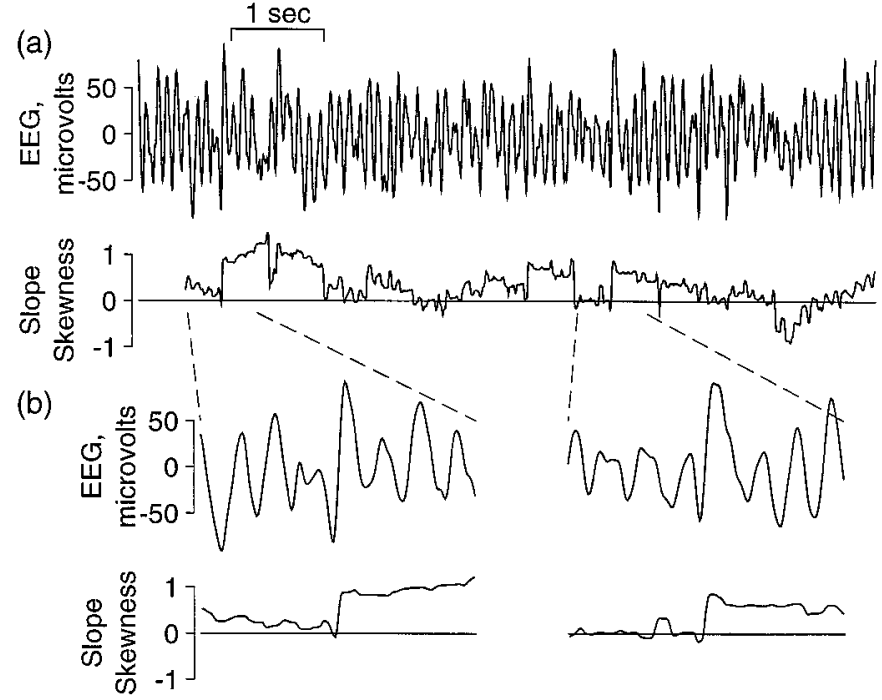

(c)

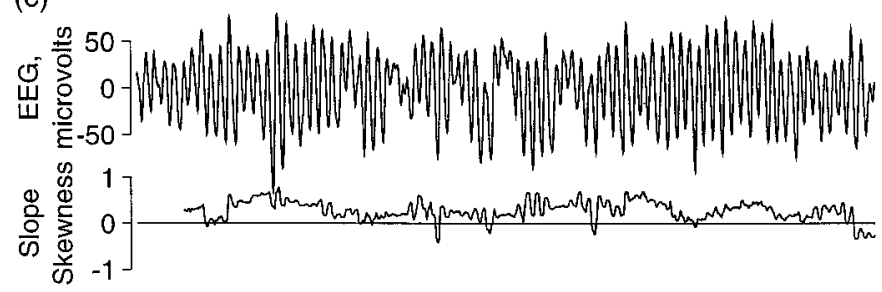

Figure 1. Slope asymmetry in the EEG. Although the slope asymmetry is computed as a single average of slope skewness (see Materials and Methods for equation), this figure illustrates how the slope skewness can vary over the time series. Two $8 \mathrm{sec}$ segments of EEG are shown in $a$ and $c$, together with the slope skewness as a moving average over the preceding $0.5 \mathrm{sec}$. In $b$, EEG and corresponding slope skewness of two brief sections from $a$ are shown with an expanded time scale, showing episodes of positive slope asymmetry in each of which a single high-amplitude EEG wave with very rapid rise, as compared with the preceding and following fall, produces an abrupt positive shift in slope skewness. A different pattern is seen in $c$, where positive skewness is more continuous, resulting from a steeper rise than fall over many EEG waves. These are representative of several observed patterns, all of which produced predominantly positive skewness of slope.

quantitative statements about this structure. When we speak informally about more or less "randomness" in the time series, we generally are referring to more or less significant evidence for nonlinearity in the data. The measures used to quantify nonlinear structure in the EEG included an estimation of correlation dimension, an inverse measure of trajectory density, a measure of determinism, information redundancy, and time series slope asymmetry. Linear aspects of the data also were quantified by using the power spectrum and the autocorrelation function. A brief conceptual description for these measures is provided below. In the following section, we will revisit each of the measures with a more detailed technical description of the methodology.

Time series slope asymmetry. Time series slope asymmetry is one of the few nonlinear measures that the eye often can detect in a data set such as the EEG, as seen in Figure 1. The concept of slope asymmetry is derived from the fact that time series generated from linear processes (such as sine waves) appear statistically the same whether the data are viewed as running forward or backward in time. By contrast, nonlinear systems such as those generated by relaxation oscillators generally have distinct rise and fall times. For such systems this measure captures the difference between the rise times (upward part) and fall times (downward part) of the oscillations in a dynamical system. One of the simplest ways to measure slope asymmetry is the skewness (third statistical moment) of slopes, as described below.

Time-delayed embedding. One of the most useful and important tools in nonlinear time series analysis is the time-delayed embedding (Packard et al., 1980). A time series data set describes the measurement of a single 
quantity (voltage on a certain electrode, for the case of EEG) as a function of time, but the underlying system generally requires several distinct variables to fully describe its state at a given instant in time; this state can be represented as a (vector-valued) point in an abstract multidimensional "state space," and the position in this state space evolves with time to trace out a "trajectory." The time-delayed embedding converts the single-quantity measurements into vector-valued quantities for which the components are time-delayed values of the Scalar time series. The important property of this embedding is that it preserves the geometric properties of this trajectory (such as its fractal dimension) and thus provides information about the dynamical properties of the system (for a rigorous mathematical justification, see Takens, 1981; Sauer et al., 1991). Many measures in this study (dimension, $1 \%$ radius, redundancy, Kaplan $\delta / \epsilon$ ) use embedded trajectories as a step toward the quantification of the behavior of nonlinear systems.

Correlation dimension. One behavior characteristic of deterministic dynamical systems is that they do not wander uniformly about the entire dynamical space but, instead, settle down (or "self-organize") into a small subspace of the full available dynamical space within which the system is evolving. This subspace is the "attractor" of the dynamical system. For chaotic systems the attractor is sometimes fractal or "strange," but for deterministic systems it is of lower dimension than the full-state space. This lower dimension indicates the number of active degrees of freedom or, approximately, the number of variables required to model the dynamics on the attractor. The correlation dimension (Grassberger and Procaccia, 1983) is one of the most popular ways to estimate the attractor dimension directly from time delay embedded data. Stochastic systems do not settle onto low-dimensional attractors, so estimating dimension is a direct way of distinguishing stochastic from deterministic systems. However, although dimension estimation is appealing conceptually, it is in practice a method fraught with peril (Theiler, 1990; Rapp, 1993).

The correlation integral is the first step in the estimation of a correlation dimension. Although the dimension is the more physically meaningful quantity, the correlation integral itself provides a more direct and reliable comparative measure. It is a positive measure, always less than or equal to one, which counts the fraction of pairs of points for which the distance between the two members is less than a certain value.

One percent radius. Instead of counting pairs of points that are a specified distance apart, the $1 \%$ radius is the smallest distance that is still larger than the smallest $1 \%$ of pairwise distances. Because points generally are clustered more closely in the dynamical space of nonrandom systems, their $1 \%$ radius is smaller than that of random systems.

Redundancy. Redundancy measures how much duplication of information occurs in a set of measurements. The process of self-organization of a dynamical system into repeatable and distinct forms produces redundancy. A purely random system that is wandering through dynamical space only repeats its travels by chance. Subsequent measurements for such a system always provide fresh information about the state of the system. For nonrandom systems the measurements made in the past provide information that permits approximate and/or probabilistic predictions of the future state of the system. For these systems each measurement adds only partial information to what was already predictable from the past; the measurements, collectively, exhibit a measure of redundancy.

Kaplan's $\delta / \epsilon$. The most straightforward way to identify determinism is to predict the future from information obtained from the past and then to wait for the future and see how well the prediction worked. For example, Scott and Schiff (1995) looked at the predictability of interictal spikes in epileptic EEGs, and Schiff et al. (1996) used mutual nonlinear prediction to characterize the coupling in neural ensembles. This approach, however, is highly sensitive to the actual strategy used for making the predictions in the first place. Thus measures based on prediction error were not used in this study. However, Kaplan (1994) has described a measure that produces a relatively direct measure of "determinism" without invoking actual predictions. This measure is based on the idea that if two points are close together on a trajectory, the images of the points at some short time later are more likely to be close together if the system is deterministic than if it is not.

\section{Technical description of the data analyses}

Segmentation of the time series. To reduce the effects of possible nonstationarity of EEG time series, we divided each 3 min EEG record into 22 $8 \mathrm{sec}$ segments, with nominal start times at intervals of $8 \mathrm{sec}$. This segment length was chosen as a compromise between the need for short segments, within which the EEG is likely to be approximately stationary, and the need for long enough segments to produce larger sample size for improved statistics, particularly with respect to adequately populating embedded system trajectories. The number of samples in each segment (2048) is a power of two to simplify the implementation of the fast Fourier transform algorithm.

Fourier transforms of finite segments of the EEG, used in the formation of surrogate time series, can introduce spurious high-frequency content. Because the transform treats the segment as one period of a periodic time series (i.e., as if the time series were an infinite number of repetitions of the finite segment), a discontinuity between the ends of the segment is treated as an instantaneous "jump" in the data, which contributes high-frequency content to the Fourier power spectrum. This high frequency appears in the surrogate data sets not as a single discontinuity but as an overall crinkliness that is not present in the original EEG. To minimize this effect, we adjusted the starting point of each segment so that the first few samples of the segment most closely match the first few samples after the segment in the original EEG time series. The discontinuity $d_{\mathrm{i}}$ was calculated for each point in the two seconds (512 sample times) starting with the nominal start point of an $8 \mathrm{sec}$ segment:

$$
d_{\mathrm{i}}=\sum_{j=0}^{4}\left(x_{i+j}-x_{i+j+n}\right)^{2} \quad i=0,1, \ldots, 511,
$$

where $i$ is the index of the initial sample of the time series $x_{\mathrm{i}}$ relative to the nominal start point, and $n$ is 2048, the number of samples in an $8 \mathrm{sec}$ time series. The start point $i$ is chosen to minimize this measure $d_{\mathrm{i}}$. Each $8 \mathrm{sec}$ EEG segment was normalized to zero mean and SD of one, with preservation of the scale factors so that the original EEG amplitudes could be regenerated. Except for power spectra, all subsequent processing used the normalized time series. Each EEG $8 \mathrm{sec}$ segment also was low-pass-filtered digitally at $45 \mathrm{~Hz}$ to reduce muscle artifact while retaining structure related to the EEG. Because this digital filtering is linear, it cannot introduce nonlinear structure into data that are not already nonlinear.

\section{Linear measures}

Of the various measures calculated in this study, those that are sensitive only to linear structure in time series are designated linear measures. The linear content of a time series is described completely by the power spectrum or, equivalently, by the autocorrelation function.

Power spectrum. The power spectrum, as opposed to other derived measures in this study, is based on the full un-normalized EEG signal and thus reflects differences in EEG amplitude among segments and subjects. The total power in each power spectrum was calculated by summing all components, and the powers in $\theta$ - and $\alpha$-bands were calculated by summing components in bands from 4 to $8 \mathrm{~Hz}$ and from 8 to 12 $\mathrm{Hz}$, respectively. $\alpha$ - and $\theta$-bands were selected because they have been demonstrated in previous studies (see Lukas et al., 1986; Ehlers et al., 1989) to differentiate most clearly the EEG after placebo from that after ethanol. The power in each band was divided by the total power to provide the $\theta$-fraction and $\alpha$-fraction. For the Fourier transforms used in the calculation of power spectra and in the preparation of surrogate time series, rectangular windowing was used.

Autocorrelation function. The autocorrelation function of each EEG and surrogate time series was calculated as the inverse Fourier transform of the power spectrum of the time series. Autocorrelation time was calculated as the autocorrelation lag at which the autocorrelation function first decreases to $1 / e$. Coherence time is defined as the lag at which the envelope of the peaks of the autocorrelation function decreases to $1 / e$ (that is, to $\sim 37 \%$ of its initial amplitude), estimated by a least-squares fit of a straight line to the logarithm of the magnitude of each positive or negative peak for lags of not more than $500 \mathrm{msec}$.

\section{Nonlinear measures}

Surrogates. Surrogates were generated by the amplitude-adjusted phaserandomized algorithm described in Theiler et al. (1992). The generation of surrogates uses the following steps: (1) An "amplitude-adjusted" copy of the EEG is prepared by means of a static nonlinear transform so that the time series has a Gaussian distribution of amplitudes; (2) the Fourier transform is calculated; (3) the transform is converted to polar form, phase angles are replaced by random numbers, and the result is converted back to complex form; (4) the inverse Fourier transform is calculated; and (5) a static nonlinear transform is used to convert to the 


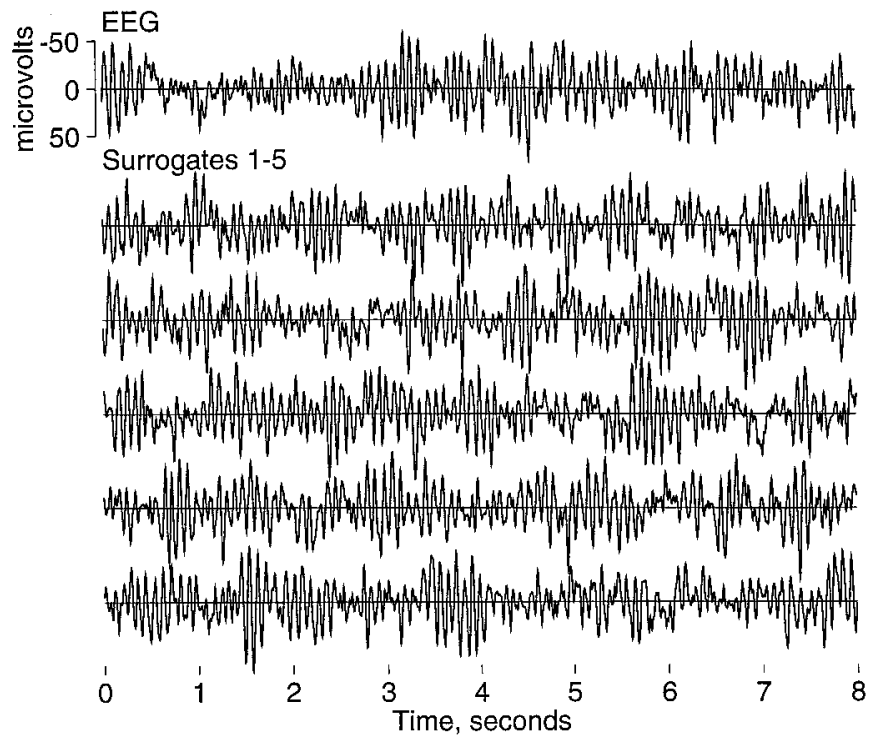

Figure 2. EEG and surrogate data. This figure displays a sample $8 \mathrm{sec}$ EEG epoch in the top trace and five surrogate time series generated from it in the traces below. Because the linear structure of the time series is preserved in surrogates, the original time series and its surrogates appear similar by visual inspection. This EEG is representative of the posterior dominant rhythm of $\alpha$-activity typical of eyes-closed human EEG. Other patterns with more or less $\alpha$-activity also were observed.

original distribution of amplitudes. The result is a surrogate time series that uses the original EEG samples but in a different sequence, with the constraint that the power spectrum and therefore the linear structure are preserved, whereas nonlinear structure in a statistical sense is removed. Figure 2 shows one such EEG segment and five of its surrogates.

Slope asymmetry. The asymmetry of the distribution of the first time derivative of each EEG or surrogate time series is estimated by the skewness of differences between successive samples,

$$
\text { Slope asymmetry }=\frac{\sum\left(x_{i}-x_{i-1}\right)^{3}}{\left(\sum\left(x_{i}-x_{i-1}\right)^{2}\right)^{3 / 2}} .
$$

Embedding. With the use of a standard algorithm (see Packard et al., 1980) containing an embedding dimension $m$ and a delay time $\tau$, the embedded vector is:

$$
x_{\tau}=\left[x_{t}, x_{t+\tau}, \ldots, x_{t+(m-1) \tau}\right] .
$$

For a deterministic system the embedding dimension should be larger than the dimension of the attractor (Takens, 1981; Sauer et al., 1991) to characterize the deterministic dynamics fully. However, evidence for determinism is often available with a lower embedding dimension, and in general the required/optimal embedding dimension is rarely known a priori. As a consequence, this study uses several values of embedding dimension. The embedding delay time $\tau$ for this study is five sample periods $(19.53 \mathrm{msec})$, which is approximately the autocorrelation time of the EEG. Embedding dimensions $m$ are 1, 4, 8, 16, and 32.

Correlation integral. The order two correlation integral, used in the calculation of several measures, is:

$C_{2}(r, m)=\frac{1}{N} \sum_{i}\left(\sum_{j} \Theta\left(r-\left\|x_{i}-x_{j}\right\|\right)\right)$,

$$
\text { for all } i, j \text { such that }|i-j|>W,
$$

where $r$ is a distance, $m$ is the embedding dimension, $\left\|x_{\mathrm{i}}-x_{\mathrm{j}}\right\|$ is the distance between points $x_{\mathrm{i}}$ and $x_{\mathrm{j}}, N$ is the number of pairs of points, $x_{\mathrm{i}}$ and $x_{\mathrm{j}}$, used, and $\Theta$ is the Heaviside function, which has the value 1 if the expression in the inner parentheses has a value greater than zero, and zero otherwise. Summation of the Heaviside function in this equation counts the number of point pairs separated by distance less than $r$, and

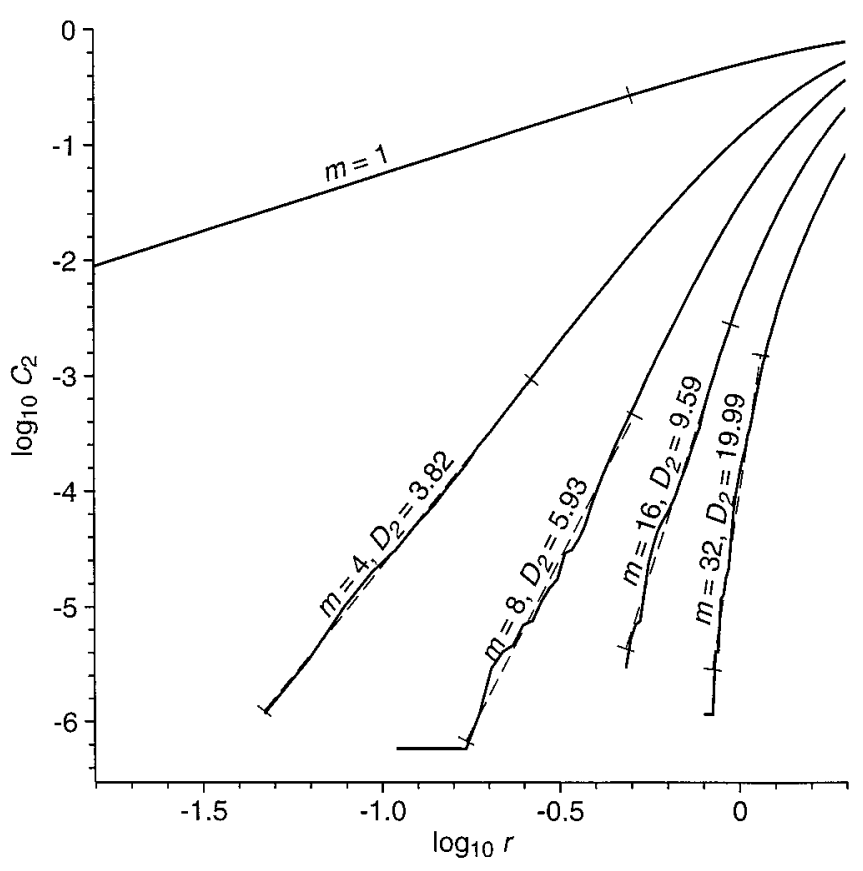

Figure 3. Log of correlation integral $C_{2}$ as a function of $\log$ of radius $r$, for various values of embedding dimension $m$. The value of the correlation integral at radius $r$ is the average fraction of all points of the trajectory lying within an $m$-dimensional cube of radius $r$, with the cube centered on a point on the trajectory. For ideal noise-free stationary low-dimensional systems, the log correlation integral should decrease linearly with decreasing log of radius at small radius; the slope of this scaling region is the correlation dimension, estimated here by the slope of the dashed straight lines fit to the curves. For curves at embedding dimension sufficiently greater than the correlation dimension, the slopes should saturate at a constant estimate of correlation dimension. The curves shown here, for a single $8 \mathrm{sec}$ segment of EEG, are representative of many that show little evidence of a low-dimensional attractor but that nevertheless have slopes less than those for surrogates of the EEG.

division by $N$ produces the correlation integral, the fraction of points separated by distance less than $r$ at embedding dimension $m$. To avoid an error caused by points closely spaced on the same orbit of the trajectory, the difference in magnitude of $i-j$ must be greater than a fixed value $W$ (Theiler, 1986) for which 50 sample periods were used. This value, corresponding to $\sim 200 \mathrm{msec}$, is greater than the $(100 \mathrm{msec})$ period of the $\alpha$-rhythm, which is the main source of trajectory periodicity. The correlation integral at embedding dimension $m$ and for radius $r$ is the fraction of pairs of points not on the same loop of the trajectory for which the separation is not greater than $r$.

The solid curves of Figure 3 are an example of correlation integral as a function of radius, for various values of embedding dimension, calculated for embedding dimension $m=1,4,8,16$, and 32, for 128 values of radius from $1 / 64$ to $2 \mathrm{SDs}$ of the time series. Maximum $\left(L_{\infty}\right)$ norm is used for the calculation of distances.

The correlation integral also is used in the calculation of the correlation dimension, $1 \%$ radius, Kaplan's $\delta / \epsilon$, and the order-two redundancy.

Correlation dimension. The correlation dimension is calculated from the correlation integral (Grassberger and Procaccia, 1983). Although the correlation dimension $D_{2}(\mathrm{~m})$ is defined in terms of a limit as radius goes to zero, it is estimated by the slope of the log of correlation integral versus log radius, as shown in Figure 3. The estimated slopes, and the range over which they are calculated, are shown as dotted lines. Slope is estimated by a least-squares fit of a straight line to points on the curve over a range of radius such that the smallest value of radius is that for which the correlation integral is greater than for the next smaller value of radius, and the largest value of radius is the smallest value for which the correlation integral is at least 1000 times its smallest value. Other methods to estimate this slope have been suggested by Takens (1981), Ellner (1988), and Theiler and Lookman (1993).

One percent radius, $\mathrm{r}_{1 \%}(\mathrm{~m})$. The radius at a particular value of corre- 
lation integral and embedding dimension is the size of an $m$-dimensional cube containing, on average, the fraction of points given by the correlation integral and is therefore an inverse measure of the density of points on the embedded trajectory. The measure $r\left(m, C_{2}\right)$ for $m=4,8,16$, and 32 , at $C_{2}=0.01$, will be called the $1 \%$ radius at embedding dimension $m$, $r_{1 \%}(m)$. The trajectories of more obviously deterministic systems are likely to be more dense, with smaller $1 \%$ radius, than those of systems in which the complexity provides less evidence of determinism.

Redundancy. Information theoretic methods in nonlinear time series analysis were advocated first by Shaw (1981) and popularized by Fraser and Swinney (1986) and Fraser (1989) over a decade ago. More recently, Palus (1995, 1996b) and Palus and colleagues (1993) have promoted the use of "redundancy" as an information theoretic measure of determinism.

One may begin with a measure of entropy $H(1, r)$, which is the average number of bits needed to describe a single value of the time series to a precision $r$. For instance, if $p_{\mathrm{r}}$ is the fraction of time series values between $x$ and $x+r$, then:

$$
\sum_{x} p_{r}=1
$$

and:

$$
H(1, r)=-\sum_{x} p_{r} \log _{2} p_{r}
$$

Let $H(m, r)$ be the average number of bits needed to describe a sequence of $m$ values (equivalently, the number of bits in an $m$-dimensional embedding $x_{\tau}$ ). In general,

$$
H(m, r) \leq m H(1, r),
$$

because describing a sequence of $m$ values cannot take more bits than describing $m$ individual values. In fact, one can usually describe the sequence with considerably fewer bits, because the time series contains some redundancy. This redundancy is defined formally by:

$$
R(m, r)=m H(1, r)-H(m, r) .
$$

The definition of entropy in the equation above for $H(1, r)$ has many useful properties, but Prichard and Theiler (1995) argue that a definition based on the correlation integral has some advantages, particularly from the viewpoint of computational accuracy from a finite set of points. Here,

$$
H_{2}(m, r)=-\log _{2}\left[C_{2}(m, r)\right],
$$

and redundancy for the embedded trajectory is:

$$
R_{2}(m, r)=m H_{2}(1, r)-H_{2}(m, r) .
$$

The range of radius over which entropy calculations are valid depends on embedding dimension, and this range may not overlap at large and small embedding dimensions. At high embedding dimension and small radius the correlation integral may be zero, resulting in infinite entropy, or large radius values at low embedding dimension may approach or exceed the size of the trajectory so that entropy is not effectively a function of radius. To avoid these cases, we calculated redundancy at embedding dimension $m$ by means of entropies at embedding dimensions $m$ and $n$, where $n$ is less than $m$, using the variant definition:

$$
R_{2}^{\prime}(m, r)=\frac{m}{n} H_{2}(n, r)-H_{2}(m, r),
$$

which reduces to the standard definition for $n=1$. For $m=16, n=4$ was used, and for $m=32, n=8$. Results are the measures $R_{2}^{\prime}(m, r)$ for $m=$ $4,8,16$, and 32 and $r=0.5,1.0,1.5$, and 2.0, of which the results are used only for those values of $r$ for which there was overlap for the stated combinations of $m$ and $n$.

\section{Kaplan's $\delta / \epsilon$}

Let the distance between two points at time $t$ be:

$$
\delta_{i, j}=\left\|x_{i}-x_{j}\right\|,
$$

where the double bars indicate the maximum norm, and let the distance between the images of the points at $k$ time steps later be:

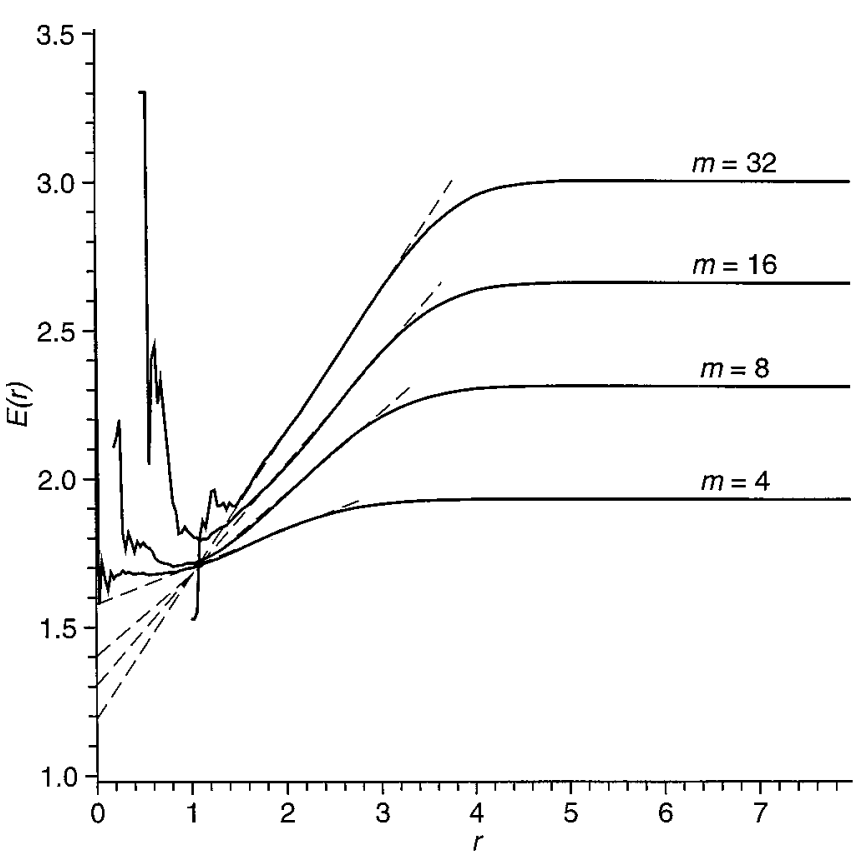

Figure 4. Kaplan's $\delta / \epsilon$ measure of determinism for a representative segment of EEG. For pairs of point pairs separated by not more than distance $r$ at time $t$, the mean separation between their images at $t+100$ $\mathrm{msec}$ is $E(r)$ for various values of embedding dimension $m$. Straight lines $E=A+B r$ are fit to the curves, as shown by the dashed lines. For completely nondeterministic systems (i.e., white noise) the slope $B$ will be close to zero, and the intercept $A$ will be close to the value of $E$ at large $r$. Decreasing values of intercept and increasing slope provide increasing evidence of deterministic structure. An ideal noise-free low-dimensional deterministic system would be expected to produce an intercept of zero, at least in the limit of infinite data, and a well defined positive slope.

$$
\epsilon_{i, j}=\left\|x_{i+k}-x_{j+k}\right\|,
$$

where $k$ is the number of sample periods in time interval $T$. For points closer together than $r$, the average separation of their images at time $T$ later is:

$E_{r}=\frac{1}{N_{r}} \sum_{i, j} \epsilon_{i, j}$ for all $i, j$ such that $|i-j|>W$, and $\delta_{i, j}<r$,

where $N_{\mathrm{r}}$ is the number of point pairs contributing to the average at the specified value of $r . E_{\mathrm{r}}$ is the average separation between all pairs $\left(x_{\mathrm{i}}, x_{\mathrm{j}}\right)$ of points that, $k$ time steps earlier, were separated by a distance $\delta_{\mathrm{i}-\mathrm{k}, \mathrm{j}-\mathrm{k}} \leq r$.

The window $W$ of excluded points is 50 , as in the calculation of the correlation integral, to avoid pairs of points on the same loop of the trajectory. The average distance $E_{\mathrm{r}}$ is calculated from the equation above for $0 \leq r<8$, accumulating sums in 256 bins of width $1 / 32$, and $k=26$ sample periods $(T=101.56 \mathrm{msec})$.

Nondeterministic systems are expected to have values of $\epsilon_{\mathrm{i}, \mathrm{j}}$ that are independent of previous separations $\delta_{i, j}$, so a plot of $E_{\mathrm{r}}$ versus $r$ will tend to be a line with zero slope. For deterministic systems, $E_{\mathrm{r}}$ is expected to be small for small $r$, increasing with increasing $r$ to a maximum that is dependent on the size of the trajectory. Figure 4 is an example of this plot. The part of the curve dependent on $r$ is characterized by a leastsquares fit of a straight line $E_{\mathrm{r}} \sim A+B r$, where $A$ is the intercept of the fit line at $r=0$, and $B$ is the slope. Points to be fit by the straight line are weighted by the number of point pairs $N_{\mathrm{r}}$ contributing to the cumulative average. All sets of consecutive points from the first nonzero point to from $1 / 6$ to $1 / 2$ of the remaining points then are tested. The set with the least residual error from the straight line fit is used as the best fit. Results are the measures identified in Table 1 as $\epsilon A(m)$, the intercept of the function at $r=0$, and $\epsilon B(m)$, the slope. Dashed lines in Figure 4 show the estimated slopes $B$ and intercept $A$ at $r=0$. 
Surrogate difference (EEG minus surrogate)

\begin{tabular}{|c|c|c|c|c|c|c|c|c|c|c|}
\hline \multirow[b]{3}{*}{ Measure } & & \\
\hline & \multicolumn{2}{|l|}{ Mean \pm SEM } & \multicolumn{2}{|l|}{$t$} & \multicolumn{2}{|l|}{$p$} & \multicolumn{2}{|l|}{$t$ test } & \multicolumn{2}{|c|}{ Rank test } \\
\hline & Placebo & Ethanol & Placebo & Ethanol & Placebo & Ethanol & Placebo & Ethanol & Placebo & Ethanol \\
\hline Slope asymmetry & $0.139 \pm 0.023$ & $0.075 \pm 0.022$ & 6.010 & 3.434 & $<0.001$ & $<0.001$ & 10.156 & 7.125 & 9.875 & 6.938 \\
\hline \multicolumn{11}{|c|}{ Correlation dimension at embedding dimension $m$} \\
\hline$m=4$ & $0.004 \pm 0.013$ & $-0.035 \pm 0.011$ & 0.282 & -3.128 & n.s. & $<0.005$ & 0.812 & 1.375 & 0.781 & 1.375 \\
\hline$m=8$ & $-0.068 \pm 0.043$ & $-0.100 \pm 0.041$ & -1.583 & -2.448 & n.s. & $<0.02$ & 1.312 & 1.094 & 1.500 & 1.219 \\
\hline$m=16$ & $-0.218 \pm 0.091$ & $-0.225 \pm 0.072$ & -2.390 & -3.120 & $<0.05$ & $<0.005$ & 1.062 & 1.250 & 1.344 & 1.344 \\
\hline$m=32$ & $-0.594 \pm 0.172$ & $-0.576 \pm 0.146$ & -3.454 & -3.932 & $<0.002$ & $<0.001$ & 1.000 & 1.188 & 1.531 & 1.094 \\
\hline \multicolumn{11}{|c|}{ One percent radius at embedding dimension $m$} \\
\hline$m=4$ & $-0.112 \pm 0.006$ & $-0.108 \pm 0.007$ & -18.632 & -16.507 & $<0.001$ & $<0.001$ & 8.562 & 7.344 & 6.812 & 5.750 \\
\hline$m=8$ & $-0.125 \pm 0.007$ & $-0.120 \pm 0.008$ & -18.632 & -15.916 & $<0.001$ & $<0.001$ & 11.656 & 10.250 & 9.969 & 8.594 \\
\hline$m=16$ & $-0.136 \pm 0.008$ & $-0.128 \pm 0.009$ & -18.075 & -14.710 & $<0.001$ & $<0.001$ & 13.906 & 12.500 & 12.531 & 10.969 \\
\hline$m=32$ & $-0.140 \pm 0.008$ & $-0.133 \pm 0.011$ & -16.771 & -12.283 & $<0.001$ & $<0.001$ & 14.219 & 12.219 & 12.562 & 11.188 \\
\hline \multicolumn{11}{|c|}{ Redundancy at embedding dimension $m$ and radius $r$} \\
\hline$m=4, r=0.5$ & $0.203 \pm 0.011$ & $0.174 \pm 0.014$ & 19.270 & 12.741 & $<0.001$ & $<0.001$ & 11.750 & 9.500 & 11.250 & 9.031 \\
\hline$m=4, r=1.0$ & $0.113 \pm 0.006$ & $0.098 \pm 0.009$ & 18.197 & 11.110 & $<0.001$ & $<0.001$ & 9.312 & 8.656 & 10.250 & 8.344 \\
\hline$m=8, r=1.0$ & $0.414 \pm 0.020$ & $0.370 \pm 0.025$ & 20.376 & 15.065 & $<0.001$ & $<0.001$ & 11.688 & 10.594 & 12.125 & 10.312 \\
\hline$m=8, r=1.5$ & $0.204 \pm 0.012$ & $0.188 \pm 0.016$ & 16.840 & 11.588 & $<0.001$ & $<0.001$ & 8.188 & 8.219 & 9.438 & 9.031 \\
\hline$m=16, r=1.5$ & $0.204 \pm 0.013$ & $0.201 \pm 0.014$ & 15.316 & 14.498 & $<0.001$ & $<0.001$ & 8.062 & 7.250 & 7.469 & 6.656 \\
\hline$m=16, r=2.0$ & $0.094 \pm 0.007$ & $0.093 \pm 0.009$ & 14.038 & 10.695 & $<0.001$ & $<0.001$ & 6.188 & 5.875 & 6.656 & 6.156 \\
\hline$m=32, r=1.5$ & $0.420 \pm 0.036$ & $0.403 \pm 0.034$ & 11.656 & 12.038 & $<0.001$ & $<0.001$ & 3.781 & 3.031 & 3.906 & 3.219 \\
\hline$m=32, r=2.0$ & $0.192 \pm 0.017$ & $0.193 \pm 0.018$ & 11.138 & 10.892 & $<0.001$ & $<0.001$ & 4.906 & 4.406 & 4.656 & 3.969 \\
\hline \multicolumn{11}{|c|}{ Cumulative $\epsilon$ intercept $A$ at embedding dimension $m$} \\
\hline$m=4$ & $-0.158 \pm 0.008$ & $-0.154 \pm 0.010$ & -19.799 & -15.214 & $<0.001$ & $<0.001$ & 12.906 & 11.250 & 12.156 & 10.719 \\
\hline$m=8$ & $-0.208 \pm 0.012$ & $-0.209 \pm 0.014$ & -17.174 & -14.936 & $<0.001$ & $<0.001$ & 9.906 & 8.906 & 10.406 & 9.562 \\
\hline$m=16$ & $-0.220 \pm 0.014$ & $-0.214 \pm 0.017$ & -15.301 & -12.647 & $<0.001$ & $<0.001$ & 9.562 & 7.312 & 8.438 & 7.781 \\
\hline$m=32$ & $-0.197 \pm 0.013$ & $-0.196 \pm 0.016$ & -14.825 & -12.170 & $<0.001$ & $<0.001$ & 7.000 & 6.094 & 5.031 & 4.438 \\
\hline \multicolumn{11}{|c|}{ Cumulative $\epsilon$ slope $B$ at embedding dimension $m$} \\
\hline$m=4$ & $0.047 \pm 0.003$ & $0.046 \pm 0.003$ & 14.071 & 18.000 & $<0.001$ & $<0.001$ & 7.250 & 6.906 & 7.750 & 6.594 \\
\hline$m=8$ & $0.060 \pm 0.004$ & $0.061 \pm 0.003$ & 13.751 & 18.358 & $<0.001$ & $<0.001$ & 4.625 & 4.375 & 7.188 & 6.125 \\
\hline$m=16$ & $0.058 \pm 0.004$ & $0.054 \pm 0.004$ & 13.687 & 13.261 & $<0.001$ & $<0.001$ & 5.281 & 4.219 & 5.656 & 4.906 \\
\hline$m=32$ & $0.039 \pm 0.003$ & $0.040 \pm 0.004$ & 11.857 & 11.115 & $<0.001$ & $<0.001$ & 3.781 & 3.938 & 3.031 & 2.656 \\
\hline
\end{tabular}

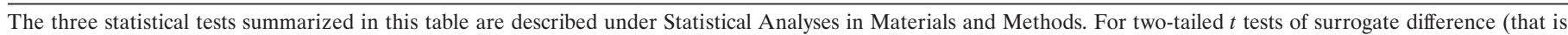

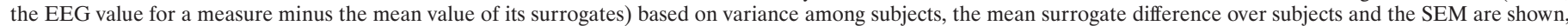

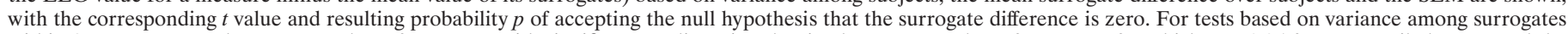

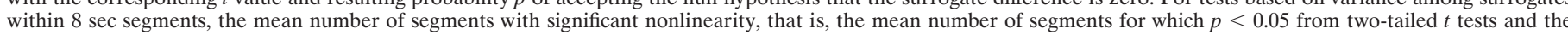

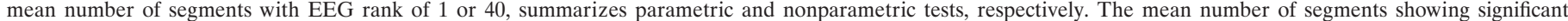

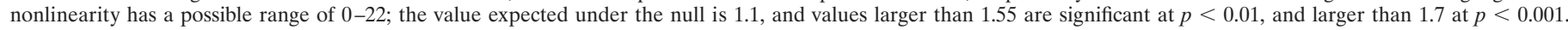

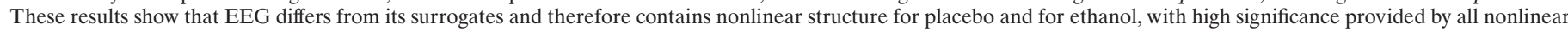

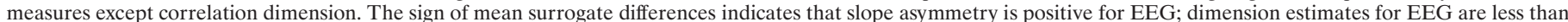

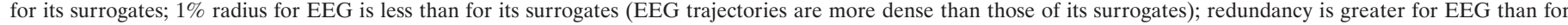

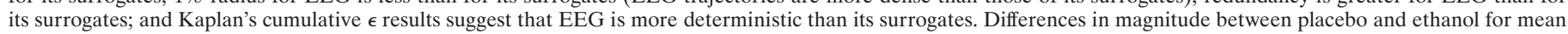
surrogate difference and mean number of segments meeting criterion suggest that EEG nonlinear structure is greater for placebo than for ethanol.

\section{Statistical analyses}

Statistical tests are described with respect to questions that they are intended to examine.

(1) Is there evidence for significant nonlinear structure in the EEG? This question was examined by comparing EEG data and their surrogates, using parametric and nonparametric tests. Because surrogates lack nonlinear structure that may be present in the EEG from which the surrogates are created, statistical tests for comparison of EEG values with those of its surrogates, for measures sensitive to nonlinear structure, serve as indicators of nonlinear structure in the EEG. Such tests require special consideration because variation among subjects, variation among $8 \mathrm{sec}$ EEG segments for each subject, and variation among surrogates for each $8 \mathrm{sec}$ segment provide separate contributions to the total variance. To avoid the pooling of these variances, we conducted three tests: two (one nonparametric and one parametric) based on variance among surrogates and one (parametric) based on variance among subjects.

The nonparametric rank-based test calculates the rank of the EEG value for each measure with respect to the combined EEG and its 39 surrogate values for each of $228 \mathrm{sec}$ segments for each subject and condition. The rank is a number in the range 1-40. The null hypothesis that there is no difference between the EEG and its surrogates can be rejected with a confidence level of $95 \%$ if the EEG rank is 1 or 40 (see Barnard, 1963; Hope, 1968). A corresponding parametric test uses the mean difference between the EEG and its surrogates, and the SD of this mean difference, over the 39 surrogates of the EEG to calculate a $t$ value and corresponding probability in a two-tailed Student's $t$ test for the same null hypothesis as for the nonparametric test. For each test the number of 8 sec segments meeting the criterion $p<0.05$ is counted and averaged over subjects, yielding the mean number of "significantly nonlinear" segments per subject, with a range of $0-22$. The third test provides an overall probability value for each measure and condition by calculating an overall mean surrogate difference and the SE of this mean difference, from which the $t$ statistic and its corresponding two-tailed $p$ value are calculated. The overall mean surrogate difference is the mean over subjects of the subject mean surrogate differences, which are averages of the $228 \mathrm{sec}$ segment mean surrogate differences for each subject. Be- 
cause this test uses only the variance among subjects, it has 31 degrees of freedom.

(2) Do EEGs collected under a placebo condition differ from those collected after ingestion of alcohol? To test this hypothesis, we used paired $t$ tests to compare data from the ethanol condition with the placebo condition for each of the measures in each subject.

(3) Are nonlinear statistics better than linear statistics for distinguishing between EEGs collected under the placebo and ethanol conditions? Discriminant function analyses were used to test this hypothesis.

(4) What measures of nonlinear structure are better in distinguishing an EEG from its surrogates or in distinguishing placebo from ethanol? Discriminant function analyses were used for this purpose, predicting membership in two mutually exclusive groups (ethanol vs placebo, or EEG vs surrogates) from a set of linear and nonlinear measures that were used as predictors.

(5) Does ethanol produce increases or decreases in evidence for nonlinear structure as compared with placebo? For this test the variable analyzed for each measure was the number for each subject of $8 \mathrm{sec}$ segments meeting the criterion that the EEG rank with respect to combined values of the EEG and its surrogates was 1 or 40 . The values for this variable were used in paired Wilcoxon signed rank tests, with the pairing of placebo and ethanol values for each subject. This nonparametric procedure avoids assumptions regarding the distribution of variables.

(6) Do linear or nonlinear measures of the actions of alcohol on the EEG correlate with a person's subjective report of intoxication? Forward selection stepwise regression was used to compare values obtained on the SHAS to linear and nonlinear EEG measures concurrently obtained during the alcohol session. Significance on this test was set at $p<0.01$.

\section{RESULTS}

\section{The EEG contains significant evidence of nonlinear structure}

The results from the three statistical tests for differences between the EEG and its surrogates are shown in Table 1. For surrogate differences (EEG values minus those of its surrogates), the $p$ values indicate that both placebo and ethanol EEGs differ with high confidence from surrogates for all nonlinear measures except correlation dimension. Because the difference between the EEG and its phase-randomized amplitude-adjusted surrogates is that the latter lack nonlinear structure, it is concluded that the EEG must contain such structure. The null hypothesis underlying the use of this type of surrogates is that the EEG is linearly filtered Gaussian noise to which a nonlinear static transform may have been applied. Results from these tests indicate, therefore, that the EEG is not modeled effectively as linearly filtered Gaussian noise.

Under the null hypothesis the expected number per subject of significantly nonlinear (using the traditional $p<0.05$ criterion) 8 sec segments is $0.05 \times 22=1.1$, and one-sided critical values for $p<0.01$ and $p<0.001$ are 1.55 and 1.7 , respectively. It is shown in Table 1 that the mean number of significantly nonlinear segments corresponds to $p<0.001$ for all nonlinear measures except correlation dimension; this holds for both parametric and nonparametric tests and for both placebo and for ethanol. Correlation dimension fails to achieve significance at any embedding dimension for placebo or for ethanol. These tests, based on variation among surrogates, are consistent with the test on the basis of variance among subjects in providing evidence for nonlinear EEG structure.

Mean numbers of significantly nonlinear segments with the conservative nonparametric test are only slightly smaller than for the parametric test. This indicates that distributions among surrogates are not unusual and that the assumptions of the $t$ test are reasonable for these data. Interestingly, the mean number of significantly nonlinear segments provides inconsistent advice regarding the difficult question of optimum embedding dimension.
For $1 \%$ radius the greatest evidence for nonlinear structure is seen at the highest embedding dimension, but for redundancy and Kaplan's cumulative $\epsilon$ measures the evidence is greatest at the lowest embedding dimension.

\section{Slope asymmetry}

Mean surrogate difference for slope asymmetry is shown in Table 1. It was found to be significant and positive for both placebo and ethanol. Figure 1 shows representative EEG waveforms and corresponding slope asymmetry in which asymmetry is seen to be dominantly positive. Slope asymmetry values for surrogates were found to be consistently near zero, providing confirmation of negligible nonlinear structure in surrogates.

\section{Correlation dimension}

The mean number of significantly nonlinear segments for correlation dimension, in Table 1 , are not notably greater than expected if the surrogate difference were zero but are sufficient to provide statistical significance of the mean surrogate difference for ethanol and for placebo at high embedding dimension. The negative values of surrogate difference indicate that estimates of trajectory dimension for surrogates are greater than for the EEG. The slopes of correlation integral curves, in Figure 3, are consistent with these results in not providing evidence for lowdimensional attractors.

\section{One percent radius}

The radius of cubes containing $1 \%$ of points is seen from surrogate difference to be greater for surrogates than for the EEG at all values of embedding dimension, indicating that EEG trajectories are denser than those of its surrogates.

\section{Redundancy}

The mean surrogate difference for redundancy was found to be positive at all values of embedding dimension and radius for both placebo and for ethanol, indicating that redundancy for the EEG is greater than for its surrogates. The mean number of significantly nonlinear segments was found to be the greatest at smallest radius at each value of embedding dimension.

\section{Kaplan's $\delta / \epsilon$}

The surrogate difference for cumulative $\epsilon$ intercept $A$ is negative and for slope $B$ is positive at all embedding dimension values, indicating that the EEG is more deterministic than its surrogates for both placebo and ethanol.

\section{The effects of ethanol can be distinguished from placebo by many measures}

The EEG after placebo could be distinguished from that after the ingestion of a low dose of ethanol by most of the EEG measures, with and without surrogates. The measures that significantly differentiated ethanol from placebo are illustrated in Figure 5. Of five linear measures the coherence time, $\alpha$-fraction, and $\theta$-fraction, but not autocorrelation time or total power, were found to differentiate ethanol significantly from placebo. Among the nonlinear measures 16 of 25 were significant, with correlation dimension at any embedding dimension and redundancy at embedding dimension of 4 and 8 failing to make the differentiation. In the matter of optimum embedding dimension, on the basis of the significance of these tests, the choice among those used would probably be $m=16$. 

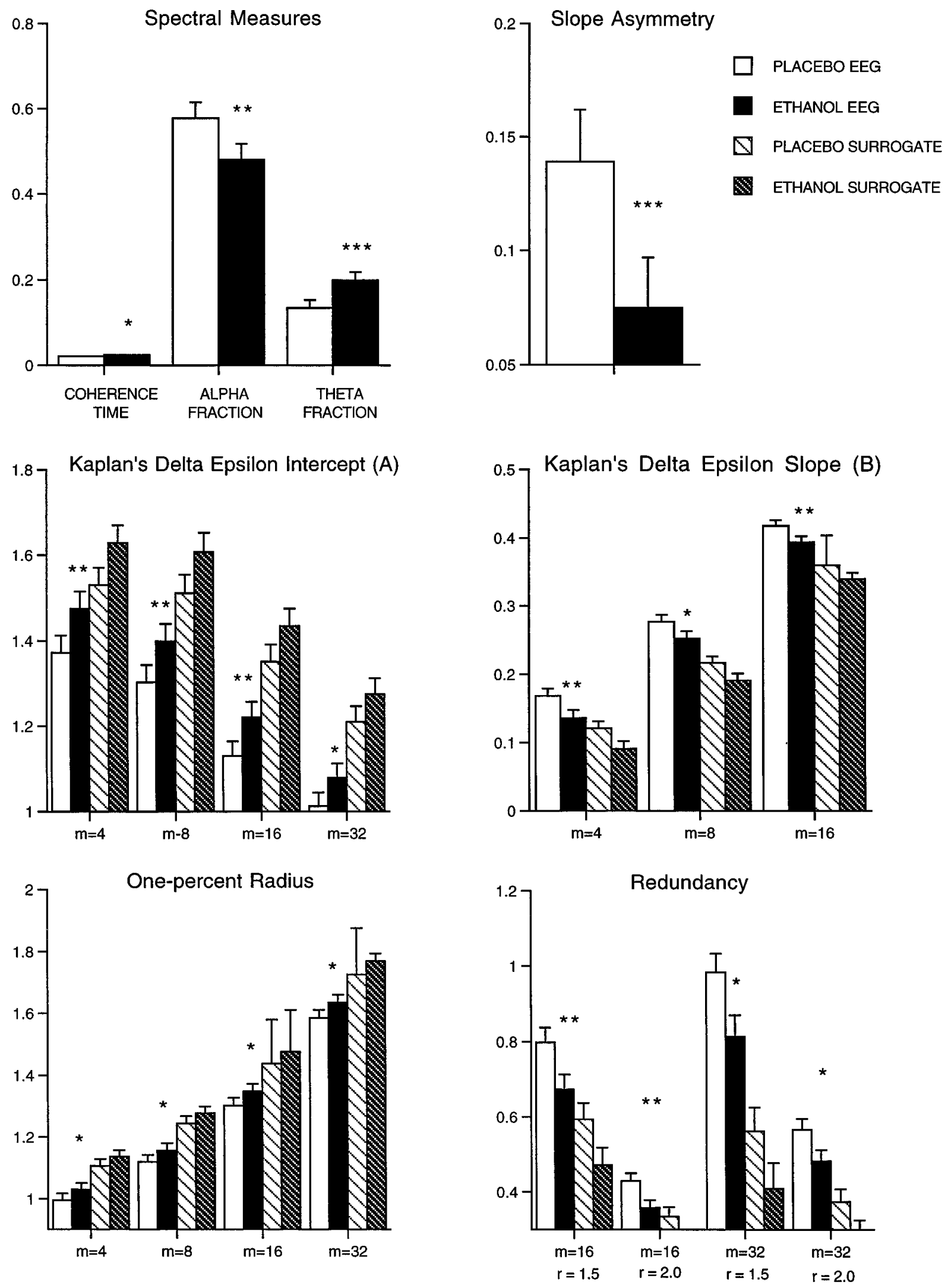

Figure 5. The effects of ethanol on the EEG. The EEG that follows placebo could be distinguished clearly from that after the ingestion of a low dose of alcohol by most of the measures. The measures that significantly differentiated ethanol from placebo are illustrated in this figure. White bars, Placebo; black bars, ethanol; lined white bars, placebo surrogates; lined black bars, ethanol surrogates. Three of five linear measures were able to distinguish ethanol from placebo. Among the nonlinear measures, 16 of 25 were significant. ${ }^{*} p<0.01 ;{ }^{* *} p<0.001 ;{ }^{* * *} p<0.0001$; paired $t$ tests. 
Table 2. Discriminant function analysis: EEG versus surrogate: nonlinear measures model (jackknifed classification matrix)

\begin{tabular}{llcl} 
& $\begin{array}{l}\text { EEG } \\
(n=32)\end{array}$ & $\begin{array}{l}\text { Surrogate } \\
(n=32)\end{array}$ & $\begin{array}{l}\text { Total correctly } \\
\text { classified }(\%)\end{array}$ \\
\hline $\begin{array}{l}\text { Number classified as EEG } \\
\text { Number classified as sur- }\end{array}$ & 28 & 0 & \\
$\quad$ rogate & 4 & 32 & \\
Correctly classified $(\%)$ & 88 & 100 & 94
\end{tabular}

A discriminant function analysis was performed to determine if a combination of nonlinear measures could describe most of the variance in nonlinear structure in the EEG. The model selected: slope asymmetry, Kaplan's $\delta \epsilon$ slope at embedding 16 , Kaplan's $\delta \epsilon$ slope at embedding 4 , and $1 \%$ radius at embedding dimension $m=4$ as the best combination to discriminate EEG from its surrogates. The classification matrix is provided in this figure; the model was found to be significant (Wilks $\lambda F=$ 32.2 , df $=1.59 ; p<0.00001$ )

\section{Multivariate analysis identifies measures of asymmetry, trajectory density, and determinism as the most able to detect nonlinear structure in the EEG}

A discriminant function analysis was used to determine whether a combination of nonlinear measures could account for most of the variance in nonlinear structure in the EEG. Mean values for each of the nonlinear measures for surrogates and the EEG were entered by using a forward selection procedure, and the model selected slope asymmetry, Kaplan's $\delta / \epsilon$ slope at embedding 16 , Kaplan's $\delta / \epsilon$ slope at embedding 4 , and $1 \%$ radius at embedding dimension $m=4$ as the best combination to discriminate the EEG from its surrogates. This model was found to be significant (Wilks $\lambda F=32.2$; df $=1,59 ; p<0.00001$ ). The jackknifed classification matrix indicates that the model was $94 \%$ correct in classifying the EEG from its surrogates. The model classified four EEG segments as surrogates but classified 0 surrogates as EEG, as seen in Table 2.

\section{Multivariate analysis identifies those linear and nonlinear measures best at discriminating between ethanol and placebo}

Discriminant function analyses also were used to determine which measures could account for the largest part of the variance between EEGs collected during alcohol and placebo conditions in separate tests that used linear and nonlinear measures. Among nonlinear measures the model chose only two, both measures of redundancy: at radius of 2 and embedding 16 and at radius of 1 and embedding 4. The model was significant (Wilks $\lambda F=5.11$; $\mathrm{df}=2,61 ; p<0.009)$, with a jackknifed classification rate of $66 \%$ correct. Of linear measures the model selected only $\theta$ fraction, which was also significant (Wilks $\lambda F=6.87$; df $=1,62 ; p<0.01$ ), and correctly classified $66 \%$ of the variance. When both linear $(\theta$ fraction) and nonlinear measures (redundancy) were combined, the resultant model was not significant at the $p<0.01$ level (Wilks $\lambda F=3.56$; df $=3,60 ; p<0.02$ ) although it classified $64 \%$ correctly, as seen in Table 3 . Although both of these models are, strictly speaking, significant, the classification rates are only slightly better than the $50 \%$ that would be expected by chance.

\section{Ethanol reduces evidence for nonlinear structure in the EEG}

To test whether ethanol produced an increase or a decrease in nonlinear structure in the EEG, we compared the evidence for nonlinearity under the placebo condition with that for the alcohol condition. This was accomplished by comparing the results for the nonparametric rank test of EEG-surrogate differences for the ethanol and placebo condition, using the Wilcoxon paired sam-
Table 3. Discriminant function model for ethanol versus placebo: nonlinear measures model (jackknifed classification matrix)

\begin{tabular}{llll} 
& $\begin{array}{l}\text { Ethanol } \\
(n=32)\end{array}$ & $\begin{array}{l}\text { Placebo } \\
(n=32)\end{array}$ & $\begin{array}{l}\text { Total correctly } \\
\text { classified }(\%)\end{array}$ \\
\hline Number classified as ethanol & 19 & 9 & \\
Number classified as placebo & 13 & 23 & \\
Correctly classified $(\%)$ & 59 & 72 & 66
\end{tabular}

Spectral measures model (jackknifed classification matrix) Ethanol Placebo Total correctly $(n=32) \quad(n=32) \quad$ classified $(\%)$

\begin{tabular}{llrl}
\hline Number classified as ethanol & 17 & 7 & \\
Number classified as placebo & 15 & 25 & \\
Correctly classified (\%) & 53 & 78 & 66
\end{tabular}

Correctly classified $(\%) \quad 53 \quad 78 \quad 66$

\begin{tabular}{llll} 
Combined nonlinear/spectral (jackknifed classification matrix) \\
& $\begin{array}{c}\text { Ethanol } \\
(n=32)\end{array}$ & $\begin{array}{c}\text { Placebo } \\
(n=32)\end{array}$ & $\begin{array}{c}\text { Total correctly } \\
\text { classified }(\%)\end{array}$ \\
\hline umber classified as ethanol & 17 & 8 & \\
umber classified as placebo & 15 & 24 & \\
orrectly classified (\%) & 53 & 75 & 64
\end{tabular}

Discriminant function analyses were applied to determine which EEG variables (nonlinear, linear, combination) could be combined to describe a significant portion of the variance between the ethanol and placebo EEGs. The upper portion of the figure presents the resultant classification matrix for nonlinear variables. This model chose only two measures, both measures of redundancy, and the model was significant (Wilks $\lambda F=5.11$; df $=2,61 ; p<0.009$ ). A model also was sought to explain the variance between ethanol and placebo, using only linear measures. This model is presented in the middle part of the table. Only $\theta$ fraction was selected, and this model was also significant (Wilks $\lambda F=6.87$; $\mathrm{df}=1,62 ; p<0.01$ ). When both linear ( $\theta$ fraction) and nonlinear measures (redundancy) were combined, the resultant model was not significant; the resultant classification matrix is presented in the bottom portion of the table.

ples signed rank test. As shown in Figure 6, the placebo condition contained significantly more evidence for nonlinear structure, when compared with ethanol, as determined by this test for the following measures: slope asymmetry $(T=90 ; p<0.002)$, correlation dimension at embedding $4(T=-106 ; p<0.004)$, redundancy at embedding 4 and radius of $0.5(T=106 ; p<0.004)$ and $1.0(T=125 ; p<0.01)$, and redundancy at embedding 32 and radius of $1.5(T=115 ; p<0.01)$. That ethanol reduces evidence for nonlinear structure is also evident by an inspection of the last four columns of Table 1. The mean number of significantly nonlinear segments is larger for placebo than for ethanol for 20 of 25 measures for the $t$ tests and for 23 of 25 measures for the rank tests. (Neglecting the four correlation dimension tests, none of which identified a significant number of nonlinear segments, these numbers become 19 of 21 for the $t$ tests and 21 of 21 for the rank tests.)

\section{Nonlinear measures of the actions of alcohol on the EEG correlate with subjective reports of intoxication}

The total score from the SHAS for the alcohol session was compared with the EEG measures obtained at that same time period ( 90 min after alcohol consumption) in a forward selection stepwise manner, using regression analyses. A model that compared all of the spectral-based measures ( $\theta$-fraction, $\alpha$-fraction, total power, autocorrelation) found no significant correlations at the $p<0.01$ or $p<0.05$ levels. None of the nonlinear measures showed a correlation that was significant at the $p<0.01$ level; the most nearly significant result was for the Kaplan $\delta / \epsilon$ measure of intercept at embedding 4 and $32(F=5.164 ; p<0.013)$. However, in testing specifically whether the nonlinear structure could ac- 


\section{PLACEBO}

\section{ETHANOL}
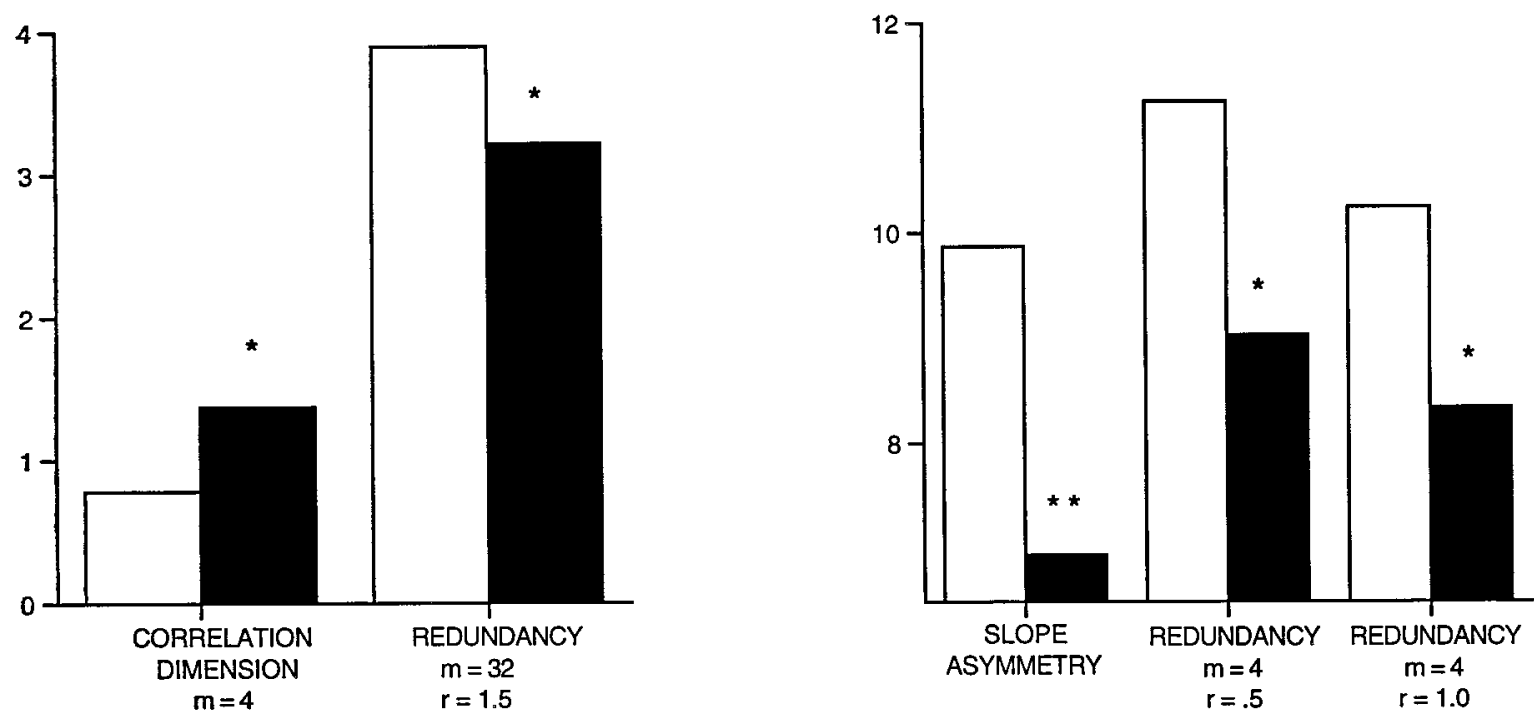

Figure 6. Ethanol reduces evidence for nonlinearity. To test whether ethanol produced an increase or a decrease in nonlinear structure in the EEG, we compared the evidence for nonlinearity under the placebo condition with that for the ethanol condition. This was accomplished by using a nonparametric rank test for each of the $8 \mathrm{sec}$ segments of EEG for each subject and comparing, for each of the nonlinear measures, the values obtained from EEG with those from its surrogates for both the placebo and ethanol conditions. Then the number of segments for which a rank of 1 or 40 was obtained for the ethanol and placebo conditions was compared, using the Wilcoxon paired samples signed rank test. The placebo condition contained significantly more evidence for nonlinear structure, as measured by slope asymmetry $(T=90 ; p<0.002)$, correlation dimension at embedding $4(T=$ $-106 ; p<0.004)$, redundancy at embedding 4 and radius of $0.5(T=106 ; p<0.004)$ and $1.0(T=125 ; p<0.01)$, and redundancy at embedding 32 and radius of $1.5(T=115 ; p<0.01) .{ }^{*} p<0.01 ; * *<0.001$.

count for a correlation between the EEG and the SHAS by using surrogate-EEG differences, we found several of the nonlinear measures to correlate significantly with the SHAS: $1 \%$ radius at embedding dimension $m=1$ and $4(F=5.2 ; p<0.01)$; Kaplan's $\delta / \epsilon$ intercept at embedding $4(F=7.1 ; p<0.01)$, and redundancy at embedding 4 and $16(F=6.7 ; p<0.002)$.

\section{DISCUSSION}

There has been considerable interest, over at least the last 15 years, in exploiting techniques developed from nonlinear dynamics for characterizing biological systems (see Mackey and Glass, 1977; Mandell, 1984; Goldberger et al., 1990; Kaplan and Cohen, 1990; Garfinkel et al., 1992; Glass and Kaplan, 1993; Lopes da Silva et al., 1994; Schiff et al., 1994a; Gottschalk et al., 1995; Kaplan and Glass, 1995; Glass, 1997). A large number of studies have focused on the evaluation of the EEG (see Rapp et al., 1989; Ehlers et al., 1991, 1995; Pijn et al., 1991; Röschke, 1992; Mann et al., 1993; Pradhan and Narayana-Dutt, 1993; Ferri et al., 1996; Stam et al., 1996) (for review, see Jansen, 1991; Pritchard and Duke, 1992). A number of authors have suggested that chaotic behavior may be present in brain electrical activity (Babloyantz et al., 1985; Rapp et al., 1985; Babloyantz and Destexhe, 1986; Skarda and Freeman, 1987; Mayer-Kress et al., 1988; Röschke and Basar, 1988; Pezard et al., 1992; Röschke and Aldenhoff, 1992, 1993; Fell et al., 1993, 1996a,b; Achermann et al., 1994a; Meyer-Lindenberg, 1996; Röschke et al., 1997), although some of these results recently have come under scrutiny (see Havstad and Ehlers, 1989; Rapp, 1993; Rapp et al., 1993; Achermann et al., 1994b; Theiler, 1995; Pritchard et al., 1996; Theiler and Rapp, 1996).
Although even simple dimension algorithms usually can estimate the dimensions of actual low-dimensional nonlinear systems, these algorithms also often report spurious low dimensions for data sets that arise from systems that are linear and/or stochastic. This difficulty has led to a renewed interest in the simpler problem of distinguishing linear from nonlinear dynamical systems. There are several aspects of nonlinear systems that can be quantified (see Theiler, 1990, 1994; Grassberger et al., 1991; Casdagli, 1992; Kantz and Schreiber, 1997). The method of surrogate data (see Kaplan and Cohen, 1990; Theiler et al., 1992; Prichard and Theiler, 1994; Rapp et al., 1994; Schreiber and Schmitz, 1996; Theiler and Prichard, 1996, 1997; Chan, 1997; Kantz and Schreiber, 1997; Schreiber, 1998) is a relatively new approach. This method compares a data set of interest with a series of surrogate data sets that are constructed in such a way as to be as "random" as possible; however, they "contain" all of the linear properties of the original data set. A comparison of original and surrogate times series, using several measures of nonlinear structure, can help to determine whether a system contains nonlinear deterministic structure rather than being simply linearly correlated noise. Using this technique, some investigators have reported no significant differences between the EEG and its surrogates (Kaplan and Cohen, 1990; Kaplan and Glass, 1992; Glass et al., 1993; Palus et al., 1993) or only small differences (Soong and Stuart, 1989; Theiler et al., 1992; Achermann et al., 1994b; Prichard and Theiler, 1994; Casdagli et al., 1997). Recently, in three studies the EEG times series was able to be distinguished from linearly filtered noise with high significance (Pritchard et al., 1995; Rombouts et al., 1995; Theiler and Rapp, 1996). However, those 
investigators also found no evidence for a low-dimensional attractor in the EEG. The present investigation confirms these findings.

Estimation of dimension was found to be one of the least sensitive measures of nonlinear structure in the EEG. The correlation integral, however, was still found to be useful for discrimination between the EEG and its surrogates as well as for several measures (trajectory density, redundancy) derived from it. The $1 \%$ radius, a measure that estimates how the points in an attractor are distributed over a section of phase space by estimating characteristic lengths or distances between points, was much more sensitive in distinguishing between the EEG and surrogates than dimension. Because many investigators rely on the estimation of dimension as a primary variable in investigating whether the EEG can be distinguished from linear filtered noise, this may be one reason that some of the early results were less than convincing. Palus (1996a) also has scrutinized the use of dimensional estimates and Lyapunov exponents in this regard.

Several measures to estimate nonlinearity that have been applied less routinely to neurophysiological systems were investigated. Kaplan's $\delta / \epsilon$ method (1994) with cumulative $\epsilon$ measure $E$ is based on the idea that, if two points are close together on a trajectory, their images at some time later are more likely to be close if the system is deterministic than if it is not. This measure was found to discriminate quite clearly between the EEG and its surrogates. Other authors have used measures of determinism to test whether simple neuronal circuits such as monosynaptic spinal cord reflexes in the cat or rat hippocampal brain slices (Chang et al., 1994; Schiff et al., 1994b; Aitken et al., 1995) display deterministic structure. Interestingly, in those studies (see Chang et al., 1994) it was found that variability in spinal cord reflexes was stochastic in an isolated spinal segment but was more deterministic if the segment was not isolated. The authors argue in their interpretation of these results that determinism in brain function may result from the integrative activity of a very large number of neurons and that the process of isolating brain parts into segments or slices may destroy, rather than enhance, determinism (see also Aitken et al., 1995; Chang et al., 1995).

Slope asymmetry provides another strikingly sensitive measure of nonlinearity (see Rothman, 1992; Tsay, 1992; van der Heyden et al., 1996). The time symmetry of linear systems is unaffected by static nonlinear transforms. Nonlinear systems can produce symmetrical waves, but according to Tong (1990), "Time irreversibility is the rule rather than the exception when it comes to nonlinearity." Slope asymmetry was found to give the strongest evidence of nonlinear structure in the EEG. Of particular interest is the finding that the eyes-closed resting EEG, which contains mainly $\alpha$-activity, in general has a positive slope asymmetry, whereas surrogates do not. Spontaneous oscillatory activity such as the spindle activity observed during barbiturate anesthesia in cats is attributed to alternating excitatory and inhibitory postsynaptic potentials (Jasper and Stefanis, 1965; Creutzfeldt et al., 1966). Episodes of nonlinear structure as measured by slope asymmetry could result from a change in relative phase between IPSPs and EPSPs.

It has been suggested that the same basic ionic systems are responsible for the emergence of all oscillations in the midfrequency range of the EEG (e.g., 7-14 Hz), including human $\alpha$-activity (Lopes da Silva et al., 1997). In in vitro studies of thalamocortical relay neurons, a $10 \mathrm{~Hz}$ oscillatory mode appears to be regulated by the level of hyperpolarization induced by synapses formed by the GABAergic neurons of the reticular nucleus and T-type calcium currents (Jahnsen and Llinás, 1984).
Wang (1994) has developed a comprehensive model of the dynamics of these thalamocortical relay neurons that is also critically dependent on the level of hyperpolarization. In addition, in a model provided by Babloyantz and Lourenco (1994), the various behavioral states of the cerebral cortex are seen as spatiotemporal "chaotic" cortical activity of increasing coherence generated by the nature of the input from the thalamus. How such models may relate to such measures of slope asymmetry or determinism as measured at the cortical surface is not known and requires further research.

Several of the measures used in this study were effective in demonstrating that EEG data collected during mild alcohol intoxication have less nonlinear structure than EEG data collected after placebo consumption. Measures of slope asymmetry and redundancy were the most sensitive to the effects of ethanol on nonlinear structure. Measures of EEG determinism, space-filling properties of the attractor, and redundancy were also predictive of the level of intoxication as reported by the subject. Linear measures of the EEG, on the other hand, failed to be predictive of the behavioral measures of intoxication. This suggests that these nonlinear EEG measures are a better measure of the effects of behaviorally relevant doses of alcohol than simple shifts in the power spectrum or autocorrelation function.

The finding that ethanol can decrease the evidence for nonlinear structure in the EEG invites speculation as to how such findings might relate to theories of the actions of ethanol on brain and behavior. Recent theories suggest that ethanol may act on both proteins and lipids in neuronal membranes (Mander et al., 1985; Besson et al., 1989; Chiou et al., 1990; Klemm, 1990; Weight, 1992; Yurttas et al., 1992; Grant, 1994; Isobe et al., 1994; Li et al., 1994; Peoples et al., 1996). How such physical changes in the cellular milieu lead to intoxication at the behavioral and electrophysiological level, especially at lower doses, has not yet been elaborated. Our finding that ethanol produces a reduction in nonlinear structure is arguably consistent with the hypothesis that ethanol may increase randomness in neuronal processing at the level of the EEG.

\section{REFERENCES}

Achermann P, Hartmann R, Gunzinger A, Guggenbühl W, Borbely AA (1994a) Correlation dimension of the human sleep electroencephalogram: cyclic changes in the course of the night. Eur J Neurosci 6:497-500.

Achermann P, Hartmann R, Gunzinger A, Guggenbühl W, Borbely AA (1994b) All-night sleep EEG and artificial stochastic control signals have similar correlation dimensions. Electroencephalogr Clin Neurophysiol 90:384-387.

Aitken PG, Sauer T, Schiff SJ (1995) Looking for chaos in brain slices. J Neurosci Methods 59:41-48.

Aston-Jones G, Foote SL, Bloom FE (1982) Low doses of ethanol disrupt sensory responses of brain noradrenergic neurones. Nature 296:857-860.

Babloyantz A, Destexhe A (1986) Low dimensional chaos in an instance of epilepsy. Proc Natl Acad Sci USA 83:3513-3517.

Babloyantz A, Lourenco C (1994) Computation with chaos: a paradigm for cortical activity. Proc Natl Acad Sci USA 91:9027-9031.

Babloyantz A, Salazar JM, Nicolis C (1985) Evidence of chaotic dynamics of brain activity during the sleep cycle. Phys Lett [A] 111:152-156.

Barnard GA (1963) Discussion on the spectral analysis of point processes by M. S. Bartlett. J R Stat Soc [B] 25:294.

Besson JA, Greentree SG, Foster MA, Rimmington JE (1989) Effects of ethanol on the NMR characteristics of rat brain. Acute administration, dependency, and long-term effects. Br J Psychiatry 155:818-821.

Casdagli MC (1992) Chaos and deterministic versus stochastic nonlinear modeling. J R Stat Soc [B] 54:303-328.

Casdagli MC, Iasemidis LD, Savit RS, Gilmore RL, Roper SN, Sackellares JC (1997) Nonlinearity in invasive EEG recordings from patients 
with temporal lobe epilepsy. Electroencephalogr Clin Neurophysiol 102:98-105.

Chan KS (1997) On the validity of the method of surrogate data. Fields Institute Commun 11:77-98.

Chang T, Schiff SJ, Sauer T, Gossard J-P, Burke RE (1994) Stochastic versus deterministic variability in simple neuronal circuits. I. Monosynaptic spinal cord reflexes. Biophys J 67:671-683.

Chang T, Sauer T, Schiff SJ (1995) Tests for nonlinearity in short stationary time series. Chaos 5:118-126.

Chiou JS, Ma SM, Kamaya H, Ueda I (1990) Anesthesia cut off phenomenon: interfacial hydrogen bonding. Science 248:583-585.

Creutzfeldt OD, Watanabe S, Lux HD (1966) Relations between EEG phenomena and potentials of single cortical cells. II. Spontaneous and convulsoid activity. Electroencephalogr Clin Neurophysiol 20:19-37.

Ehlers CL (1988) ERP responses to ethanol and diazepam administration in squirrel monkeys. Alcohol 5:315-320.

Ehlers CL (1992) The new physics of chaos: can it help us to understand the effects of alcohol? Alcohol Health Res World 16:267-272.

Ehlers CL, Havstad JW (1982) Characterization of drug effects on the EEG by power spectral band time series analysis. Psychopharmacol Bull 18:43-47.

Ehlers CL, Reed TK (1987) Ethanol effects on EEG spectra in monkeys: comparison to morphine and diazepam. Electroencephalogr Clin Neurophysiol 66:317-321.

Ehlers CL, Schuckit MA (1990) EEG fast frequency activity in the sons of alcoholics. Biol Psychiatry 27:631-641.

Ehlers CL, Schuckit MA (1991) Evaluation of EEG alpha activity in sons of alcoholics. Neuropsychopharmacology 4:199-205.

Ehlers CL, Wall TL, Schuckit MA (1989) EEG spectral characteristics following ethanol administration in young men. Electroencephalogr Clin Neurophysiol 73:179-187.

Ehlers CL, Havstad JW, Garfinkel A, Kupfer DJ (1991) Nonlinear analysis of EEG sleep states. Neuropsychopharmacology 5:167-176.

Ehlers CL, Havstad JW, Schuckit MA (1995) EEG dimension in the sons of alcoholics. Alcohol Clin Exp Res 19:992-998.

Ellner S (1988) Estimating attractor dimensions from limited data: a new method, with error estimates. Phys Lett [A] 133:128-133.

Fell J, Röschke J, Beckmann P (1993) Deterministic chaos and the first positive Lyapunov exponent: a nonlinear analysis of the human electroencephalogram during sleep. Biol Cybern 69:139-146.

Fell J, Röschke J, Schäffner C (1996a) Surrogate data analysis of sleep electroencephalograms reveals evidence for nonlinearity. Biol Cybern 75:85-92.

Fell J, Röschke J, Mann K, Schäffner C (1996b) Discrimination of sleep stages: a comparison between spectral and nonlinear EEG measures. Electroencephalogr Clin Neurophysiol 98:401-410.

Ferri R, Alicata F, Del Gracco S, Elia M, Musumeci SA, Steffanini MC (1996) Chaotic behavior of EEG slow-wave activity during sleep. Electroencephalogr Clin Neurophysiol 99:539-543.

Fraser AM (1989) Information and entropy in strange attractors. IEEE Trans Information Theory 35:245-262.

Fraser AM, Swinney HL (1986) Independent coordinates for strange attractors from mutual information. Phys Rev [A] 33:1134-1140.

Garfinkel A, Spano ML, Ditto WL, Weiss JN (1992) Controlling cardiac chaos. Science 257:1230-1235.

Glass L (1997) Dynamics of cardiac arrhythmias. Phys Today 49:40-45.

Glass L, Kaplan D (1993) Time series analysis of complex dynamics in physiology and medicine. Med Prog Technol 19:115-128.

Glass L, Kaplan DT, Lewis J (1993) Tests for deterministic dynamics in real and model neural networks. In: Proceedings of the second annual conference on EEG and nonlinear dynamics (Jansen JH, Brandt ME, eds), pp 233-249. Singapore: World Scientific.

Goldberger AL, Rigney DR, West BJ (1990) Chaos and fractals in human physiology. Sci Am 262:42-49.

Goldstein DB (1984) The effects of drugs on membrane fluidity. Annu Rev Pharmacol Toxicol 24:43-64.

Gottschalk A, Bauer MS, Whybrow PC (1995) Evidence of chaotic mood variation in bipolar disorder. Arch Gen Psychiatry 52:947-959.

Grant KA (1994) Emerging neurochemical concepts in the actions of ethanol at ligand-gated ion channels. Behav Pharmacol 5:383-404.

Grassberger P, Procaccia I (1983) Measuring the strangeness of strange attractors. Physica [D] 9:189-208.

Grassberger P, Schreiber T, Schaffrath C (1991) Nonlinear time sequence analysis. Int J Bifurc Chaos 1:521-548.
Havstad JW, Ehlers CL (1989) Attractor dimension of non- stationary dynamical systems from small data sets. Phys Rev 39:845-853.

Hope ACA (1968) A simplified Monte Carlo significance test procedure. J R Stat Soc [B] 30:582-598.

Isobe S, Hazlewood CF, Misra LK, Klemm WR (1994) Acute ethanol decreases NMR relaxation times of water hydrogen protons in fish brain. Alcohol 11:571-576.

Jahnsen H, Llinás RR (1984) Ionic basis for the electroresponsiveness and oscillatory properties of guinea-pig thalamic neurones in vitro. J Physiol (Lond) 349:227-247.

Jansen BH (1991) Quantitative analysis of electroencephalograms: is there chaos in the future? Int J Biomed Comput 27:95-123.

Jasper HH, Stefanis C (1965) Intracellular oscillatory rhythms in pyramidal tract neurones in the cat. Electroencephalogr Clin Neurophysiol 18:541-553.

Judd LL, Hubbard RP, Huey LY, Attewell PA, Janowsky DS, Takahashi KI (1977) Lithium carbonate and ethanol induced "highs" in normal subjects. Arch Gen Psychiatry 34:463-467.

Kantz H, Schreiber T (1997) Nonlinear time series analysis. London: Cambridge UP.

Kaplan DT (1994) Exceptional events as evidence for determinism. Physica [D] 73:38-48.

Kaplan DT, Cohen RJ (1990) Is fibrillation chaos? Circ Res 67:886-892.

Kaplan DT, Glass L (1992) Direct test for determinism in a time series. Phys Rev Lett 68:427-430.

Kaplan DT, Glass L (1995) Understanding nonlinear dynamics. New York: Springer.

Klemm WR (1990) Dehydration: a new alcohol theory. Alcohol 7:49-59.

Li C, Peoples RW, Weight FF (1994) Alcohol action on a neuronal membrane receptor: evidence for a direct interaction with the receptor protein. Proc Natl Acad Sci USA 91:8200-8204.

Lopes da Silva FH, Pijn JP, Wadman WJ (1994) Dynamics of local neuronal networks: control parameters and state bifurcations in epileptogenesis. Prog Brain Res 102:359-370.

Lopes da Silva FH, Pijn JP, Velis D, Nijssen PCG (1997) Alpha rhythms: noise, dynamics, and models. Int J Psychophysiol 26:237-249.

Lukas SE, Mendelson JH, Benedikt RA, Jones B (1986) EEG alpha activity increases during transient episodes of ethanol-induced euphoria. Pharmacol Biochem Behav 25:889-895.

Mackey MC, Glass L (1977) Oscillation and chaos in physiological control systems. Science 6:287-289.

Mandell AJ (1984) Non-equilibrium behavior of some brain enzyme and receptor systems. Annu Rev Pharmacol Toxicol 24:237-274.

Mander AJ, Smith MA, Dean DM, Chick J, Douglas RJB, Rehman AH, Weppner GJ, Best JJK (1985) Brain water measured in volunteers after alcohol and vasopressin. Lancet 2:1075.

Mann K, Backer P, Röschke J (1993) Dynamical properties of the sleep EEG in different frequency bands. Int J Neurosci 73:161-169.

Mayer-Kress G, Yates FE, Benton L, Keidel M, Tirsch W, Poppl SJ, Geist K (1988) Dimension analysis of nonlinear oscillations in brain, heart, and muscle. Math Biosci 90:155-182.

Mendelson JH, McGuire M, Mello NK (1984) A new device for administering placebo alcohol. Alcohol 1:417-419.

Meyer-Lindenberg A (1996) The evolution of complexity in human brain development: an EEG study. Electroencephalogr Clin Neurophysiol 99:405-411.

Packard NH, Crutchfield JP, Farmer JD, Shaw RS (1980) Geometry from a time series. Phys Rev Lett 45:712-716.

Palus M (1995) Testing for nonlinearity using redundancies: quantitative and qualitative aspects. Physica [D] 80:186-205.

Palus M (1996a) Nonlinearity in normal human EEG: cycles, temporal asymmetry, nonstationarity and randomness, not chaos. Biol Cybern 75:389-396.

Palus M (1996b) Course-grained entropy rates for characterization of complex time series. Physica [D] 93:64-77.

Palus M, Albrecht V, Dvorak I (1993) Information theoretic test for nonlinearity in time series. Phys Lett [A] 175:203-209.

Peoples RW, Weight FF (1995) Cutoff in potency implicates alcohol inhibition of $N$-methyl-D-aspartate receptors in alcohol intoxication. Proc Natl Acad Sci USA 22:2825-2829.

Peoples RW, Chaoying L, Weight FF (1996) Lipid vs protein theories of alcohol action in the nervous system. Annu Rev Pharmacol Toxicol 36:185-201. 
Pezard L, Martinerie J, Breton F, Renault B (1992) Nonlinear forecasting and correlation dimension of brain dynamics: a multichannel study. In: Proc 14th Annu Int Conf IEEE-EMBS 3:979-981.

Pijn JP, Van Neerven J, Noest A, Lopes da Silva FH (1991) Chaos or noise in EEG signals; dependence on state and brain site. Electroencephalogr Clin Neurophysiol 79:371-381.

Pradhan N, Narayana-Dutt D (1993) A nonlinear perspective in understanding the neurodynamics of EEG. Comput Biol Med 2:425-442.

Prichard D, Theiler J (1994) Generating surrogate data for time series with several simultaneously measured variables. Phys Rev Lett 73:951-954.

Prichard D, Theiler J (1995) Generalized redundancies for time series analysis. Physica [D] 84:476-493.

Pritchard WS, Duke DW (1992) Measuring chaos in the brain: a tutorial review of nonlinear dynamical EEG analysis. Int J Neurosci 67:31-88.

Pritchard WS, Duke DW, Krieble KK (1995) Dimensional analysis of resting human EEG II: surrogate data testing indicates nonlinearity but not low-dimensional chaos. Psychophysiology 32:486-491.

Pritchard WS, Krieble KK, Duke DW (1996) On the validity of estimating EEG correlation dimension from a spatial embedding. Psychophysiology 33:362-368.

Rall TW (1990) Hypnotics and sedatives: ethanol. In: The pharmacological basis of therapeutics, 8th Ed (Gilman AG, Rall TW, Nies AS, Taylor P, eds), pp 345-382. New York: Pergamon.

Rapp PE (1993) Chaos in the neurosciences: cautionary tales from the frontier. The Biologist 40:89-94.

Rapp PE, Zimmerman ID, Albano AM, de Guzman GC, Greenbaun NN, Bashore TR (1985) Experimental studies of chaotic neural behavior. In: Cellular activity and electroencephalographic signals (Othmer HG, ed), pp 175-205. New York: Springer.

Rapp PE, Bashore TR, Martinerie JM, Albano AM, Zimmerman ID, Mees AI (1989) Dynamics of brain electrical activity. Brain Topogr 2:99-119.

Rapp PE, Albano AM, Schmah TI, Farwell LA (1993) Filtered noise can mimic low-dimensional chaotic attractors. Phys Rev [E] 47:2289-2296.

Rapp PE, Albano AM, Zimmerman ID, Jimenez-Montano MA (1994) Phase-randomized surrogates can produce spurious identifications of non-random structure. Phys Lett [A] 192:27-33.

Rombouts SA, Keunen RW, Stam CJ (1995) Investigation of nonlinear structure in multichannel EEG. Phys Lett [A] 202:352-358.

Röschke J (1992) Strange attractors, chaotic behavior, and informational aspects of sleep EEG data. Neuropsychobiology 25:172-176.

Röschke J, Aldenhoff JB (1992) A nonlinear approach to brain function: deterministic chaos and sleep EEG. Sleep 15:95-101.

Röschke J, Aldenhoff JB (1993) Estimation of the dimensionality of sleep-EEG data in schizophrenics. Eur Arch Psychiatry Clin Neurosci 242:191-196.

Röschke J, Basar E (1988) The EEG is not simple noise: strange attractors in intracranial structures. In: Dynamics of sensory and cognitive processing in the brain (Basar E, ed), pp 203-216. Berlin: Springer.

Röschke J, Fell J, Mann K (1997) Nonlinear dynamics of alpha and theta rhythm: correlation dimensions and Lyapunov exponents from healthy subject's spontaneous EEG. Int J Psychophysiol 6:251-261.

Rothman P (1992) The comparative power of the TR test against simple threshold models. J Appl Econometr 7:S187-S195.

Sauer T, Yorke JA, Casdagli M (1991) Embedology. J Stat Phys 65:579-616.

Schiff SJ, Jerger K, Duong DH, Chang T, Spano ML, Ditto WL (1994a) Controlling chaos in the brain. Nature 370:615-620.

Schiff SJ, Sauer T, Chang T (1994b) Discriminating deterministic versus stochastic dynamics in neuronal activity. Integr Physiol Behav Sci 29:246-261.

Schiff SJ, So P, Taeun C, Burke RE (1996) Detecting dynamical interdependence and generalized synchrony through mutual prediction in a neural ensemble. Phys Rev [E] 54:6708-6724.

Schreiber T (1998) Constrained randomization of time series data. Phys Rev Lett 80:2105-2108.
Schreiber T, Schmitz A (1996) Improved surrogate data for nonlinearity tests. Phys Rev Lett 77:635-638.

Schuckit MA (1985) Genetics and the risk for alcoholism. JAMA 254:2614-2617.

Schuckit MA, Gold EO (1988) A simultaneous evaluation of multiple markers of ethanol/placebo challenges in sons of alcoholics and controls. Arch Gen Psychiatry 45:211-216.

Scott DA, Schiff SJ (1995) Predictability of EEG interictal spikes. Biophys J 69:1748-1757.

Seeman P (1972) The membrane actions of anesthetics and tranquilizers. Pharmacol Rev 24:583-655.

Shaw RS (1981) Strange attractors, chaotic behavior, and information flow. Z Naturforsch 36:80-112.

Skarda CA, Freeman WJ (1987) How brains make chaos in order to make sense of the world. Behav Brain Sci 10:161-195.

Soong AC, Stuart CI (1989) Evidence of chaotic dynamics underlying the human alpha-rhythm electroencephalogram. Biol Cybern 62:55-62.

Stam CJ, van Woerkom TC, Pritchard WS (1996) Use of nonlinear EEG measures to characterize EEG changes during mental activity. Electroencephalogr Clin Neurophysiol 99:214-224.

Takens F (1981) Detecting strange attractors in fluid turbulence. In: Dynamical systems and turbulence (Rand D, Young LS, eds), pp 365-381. New York: Springer.

Theiler J (1986) Spurious dimension from correlation algorithms applied to limited time-series data. Phys Rev [A] 34:2427-2432.

Theiler J (1990) Estimating fractal dimension. J Opt Soc Am [A] 7:1055-1073.

Theiler J (1994) Two tools to test time series data for evidence of chaos and/or nonlinearity. Integr Physiol Behav Sci 29:211-216.

Theiler J (1995) On the evidence for low-dimensional chaos in an epileptic electroencephalogram. Phys Lett [A] 196:335-341.

Theiler J, Lookman T (1993) Statistical error in a chord estimator of correlation dimension: the "rule of five." Int J Bifurc Chaos 3:765-771.

Theiler J, Prichard D (1996) Constrained-realization Monte Carlo method for hypothesis testing. Physica [D] 94:221-235.

Theiler J, Prichard D (1997) Using "surrogate surrogate data" to calibrate the actual rate of false positives in tests for nonlinearity in time series. Fields Institute Commun 11:99-113.

Theiler J, Rapp PE (1996) Reexamination of the evidence for lowdimensional, nonlinear structure in the human electroencephalogram. Electroencephalogr Clin Neurophysiol 98:213-222.

Theiler J, Eubank S, Longtin A, Galdrikian B, Farmer JD (1992) Testing for nonlinearity in time series: the method of surrogate data. Physica [D] 58:77-94.

Tong H (1990) Nonlinear time series: a dynamical system approach, $p$ 195. New York: Oxford UP.

Trudell JR (1977) A unitary theory of anesthesia based on lateral phase separations in nerve membranes. Biophys J 18:358-359.

Tsay RS (1992) Model checking via parametric bootstraps in time series analysis. Appl Stat 41:1-15.

van der Heyden MJ, Diks, Pijn JPM, Velis DN (1996) Time reversibility of intracranial human EEG recordings in medial temporal lobe epilepsy. Phys Lett [A] 216:283-288.

Wall TL, Thomasson HR, Schuckit MA, Ehlers CL (1992) Subjective feelings of alcohol intoxication in Asians with genetic variations of ALDH2 alleles. Alcohol Clin Exp Res 16:991-995.

Wall TL, Gallen CC, Ehlers CL (1993) Effects of alcohol on the EEG in Asian men with genetic variations of ALDH2. Biol Psychiatry 34:91-99.

Wang XJ (1994) Multiple dynamical modes of thalamic relay neurons: rhythmic bursting and intermittent phase-locking. Neuroscience 59:21-31.

Weight FF (1992) Cellular and molecular physiology of alcohol actions in the nervous system. Int Rev Neurobiol 33:289-348.

Yurttas L, Dale BE, Klemm WR (1992) FTIR evidence for alcohol binding and dehydration in phospholipid and ganglioside micelles. Alcohol Clin Exp Res 16:863-869. 